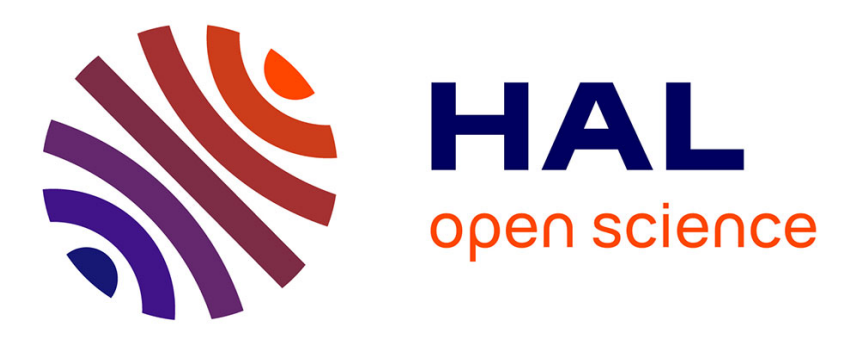

\title{
In situ $\mu$ CT-scan mechanical tests: Fast 4D mechanical identification
}

\author{
Clément Jailin, Amine A Bouterf, Martin Poncelet, Stéphane Roux
}

\section{To cite this version:}

Clément Jailin, Amine A Bouterf, Martin Poncelet, Stéphane Roux. In situ $\mu$ CT-scan mechanical tests: Fast 4D mechanical identification. Experimental Mechanics, 2017, 10.1007/s11340-017-0305-z . hal-01544539

\section{HAL Id: hal-01544539 \\ https://hal.science/hal-01544539}

Submitted on 10 Jul 2017

HAL is a multi-disciplinary open access archive for the deposit and dissemination of scientific research documents, whether they are published or not. The documents may come from teaching and research institutions in France or abroad, or from public or private research centers.
L'archive ouverte pluridisciplinaire HAL, est destinée au dépôt et à la diffusion de documents scientifiques de niveau recherche, publiés ou non, émanant des établissements d'enseignement et de recherche français ou étrangers, des laboratoires publics ou privés. 


\title{
In situ $\mu \mathrm{CT}$-scan mechanical tests: Fast 4D mechanical identification
}

\author{
C. Jailin • A. Bouterf · M. Poncelet • S. \\ Roux
}

Received: date / Accepted: date

\begin{abstract}
A recently proposed "Projection-based Digital Volume Correlation" (PDVC) method is extended in this work to a cone-beam lab-tomograph in which a mechanical test is performed. This consists of a crack propagation test in an elasticbrittle gypsum specimen. Kinematic analysis is performed based on a reduced finite element modeling for which the appropriate boundary conditions and crack propagation stage are determined from the radiographs. By considering only two projections per loading step, an integrated model-based analysis of the entire test provides a full space and time identification of the kinematics, including the crack position and the determination of two material parameters. This is achieved with a drastic reduction in the acquisition time compared to classical digital volume correlation analysis. In the examples presented, the acquisition time was reduced by a factor of 350 .
\end{abstract}

Keywords Full field measurement; DVC; P-DVC; CT-scan; in-situ mechanical test

\section{Introduction}

The identification and validation of mechanical models used to predict the behavior of materials and structures has been and still is the central focus of experimental mechanics. However, the ever increasing sophistication of mechanical models and the multiplicity of scales required to assess and quantify the microscopic phenomena at play present challenging demands to mechanical tests. During the last decades, this trend has been balanced by accessibility to increasingly powerful measuring and imaging techniques (and, to a lesser extent, richer and more accurate loading setups), from which complex loadings, specimen geometries and full-field measurements can be established.

C. Jailin et al.

LMT (ENS Paris-Saclay/CNRS/Univ. Paris-Saclay),

61 avenue du Président Wilson, F-94235 Cachan (FRANCE)

Tel.: +33 (0) 147402238

Fax: +33 (0) 147402240

E-mail: clement.jailin@ens-paris-saclay.fr 
The development of Computed Tomography (CT) has been revolutionary in materials science [1,2,3]. Accessing the intimate micro-structure of solids in a nondestructive way has opened new horizons. The recent evolutions of these imaging techniques provide access to higher spatial and temporal resolutions. First developed on synchrotron facilities with high brightness monochromatic coherent X-ray beams, X-ray CT scanners are now accessible equipment in laboratories. Moreover, their state-of-the-art performance may in favorable cases compare well with the performance of large-scale synchrotron tomography.

The development of material tests coupled with tomographic images has been studied in recent years. After ex situ testing where the materials are deformed outside of the tomograph, the recent evolution of CT makes in situ tests possible [4, 5. 6]. In the latter case, material specimens are deformed inside of the tomograph. This method allows for characterizing new mechanisms (i.e., crack opening [7,8, shear banding, and fast transformation [9, 10]). Recent works developed in synchrotron facilities reported $20 \mathrm{~Hz}$ scans for the study of crack propagation [11. Combined with imaging analysis techniques, such as full field measurement, X-ray CT becomes a powerful tool for experimental mechanics.

Digital Volume Correlation (DVC) [12,13 is a full field measurement method that aims at capturing the way a solid has deformed between two states captured in 3D images. Extended from 2D digital image correlation, DVC allows accurate measurement of $3 \mathrm{D}$ displacement fields based on the micro-structure of the registered volumes. As in standard mechanical tests, an experiment is generally composed of several loading steps. DVC can be performed with all deformed states of the same specimen. This space-time analysis of the displacement field, which is referred to as 4D-DVC [14,15], permits, for example, the identification of a constitutive law exploiting all loading steps globally.

Nevertheless, the major limitation of CT imaging, especially in lab tomographs, is the acquisition time (approximately one hour). Demanding applications requiring very high resolution can take more than 20 hours [16, 17].

This limit does not allow for the visualization of time-dependent behaviors that may be considered as artifacts, which can blur the reconstruction [18. In [19, 7], 0.5 to 1 hour was spent waiting for relaxation or creep behavior at each loading step. Another limitation is the study of biological materials where a low dose of $\mathrm{X}$-rays is used to avoid radiation-induced damage [20]. One possible procedure to circumvent these difficulties is the Projection-based DVC (P-DVC) [21]. Instead of working with full $3 \mathrm{D}$ images at every loading step, P-DVC operates directly on radiographs (i.e., projection of the deformed volume). A very small number of radiographs turns out to be sufficient to measure a displacement after a first complete 3D image of the reference state has been (classically) reconstructed. In [22,23], the measurement of the 3D displacement field of a cast iron specimen with a fatigue crack was obtained from no more than two radiographs (instead of 600). The exploited experiment had been performed in situ at the European Synchrotron Research Facility (ESRF) in Grenoble. Ideas in a similar spirit for rigid grain tracking were recently proposed in [24].

The present paper aims at extending this P-DVC methodology using lab tomograph images to address the identification of material properties from a complex geometry. In Section 2, a brief overview of the DVC and P-DVC procedures is given. This image processing technique is regularized with a coupling to a mechanical model. In Section 3, the Double Cleavage Drilled Compression (DCDC) 
test case is introduced. The proposed methodology is applied in Section 4 to the test case, and the obtained displacement fields and projection residuals are presented. The identification of model parameters is discussed. Finally, Section 6 recapitulates the main results of the paper and proposes a discussion of possible ways to improve the proposed methodology.

\section{Method}

\subsection{Digital Volume Correlation}

Digital Volume Correlation (DVC) [12,13] is a full field measurement technique for the 3D displacement field that relates two 3D images: one image for the reference state and one image for the deformed state. DVC consists of the registration of an image $f(\boldsymbol{x})$ in the reference configuration and a series of 3D images $g(\boldsymbol{x}, t)$ in the deformed configurations indexed by time $t$. The term $\boldsymbol{x}$ denotes the Cartesian coordinates of the reconstruction. The DVC procedure (written here with the Eulerian transformation to unify notations, considering the next section) is the minimization of the quadratic difference between the reference image corrected by the measured displacement $\boldsymbol{u}(\boldsymbol{x}, t)$ and the image of the deformed state (referred to as the "deformed image" for conciseness)

$$
\chi_{\boldsymbol{u}}^{2}(t)=\frac{1}{2|\Omega| \gamma_{f}^{2}} \sum_{\boldsymbol{x} \in \Omega}(f(\boldsymbol{x}-\boldsymbol{u}(\boldsymbol{x}, t))-g(\boldsymbol{x}, t))^{2}
$$

where $\gamma_{f}^{2}$ is an estimate of the noise variance over the image, and $|\Omega|$ is the volume (number of voxels) of the region of interest, $\Omega$. A first kinematic regularization of the displacement field can be introduced by global DVC. In global DVC [25], the displacement field is expressed on a reduced basis, composed of a set of fields $\Phi_{i}(\boldsymbol{x})$ such that

$$
\boldsymbol{u}(\boldsymbol{x}, t)=\sum_{i} \boldsymbol{u}_{i}(t) \Phi_{i}(\boldsymbol{x})
$$

A general framework for the kinematic bases well suited to mechanical modeling is the framework used in the finite element method. This choice offers continuous displacement fields and constitutes an ideally suited interface with finite element computations if needed, for instance, for future identification purposes.

The displacement field is finally obtained from the minimization of the functional with respect to the degrees of freedom $\boldsymbol{u}_{i}(t)$ (i.e., the nodal displacements),

$$
\boldsymbol{u}_{i}(t)=\operatorname{Argmin}_{\boldsymbol{v}_{i}}\left(\chi_{\boldsymbol{v}}^{2}(t)\right) .
$$

\subsection{Global P-DVC procedure}

To reduce the acquisition time (especially the acquisition of the entire sinogram for every deformed state), working with a few radiographs instead of reconstructed $3 \mathrm{D}$ volumes is proposed. The proposed method is Projection-based Digital Volume Correlation (P-DVC) 21,22, 23. 
The first step of the procedure is the acquisition and reconstruction of a reference image, $f(\boldsymbol{x})$ (generally without loading), from a complete set of radiographs with a fine sampling of all rotation angles. Then, the deformed states are characterized through a much reduced set of radiographs sampled at a few, $N_{\theta}$, selected angles. Because the number $N_{\theta}$ of needed projections may be very small, the deformed image cannot be reconstructed solely based on these radiographs. The idea is contrary to obtaining the deformed state from the reference image by fitting the displacement field so that the projections of the deformed volume match the few available radiographs.

To clarify our notations, recall that the reconstruction is based on images that are computed as the cologarithm of intensities of radiographs normalized by a flat field (i.e., radiograph captured without specimens). The latter are denoted as $s(\boldsymbol{r}, \theta, t)$, with $\boldsymbol{r}$ being the coordinates of the detector, $\theta$ the rotation angle and $t$ time. To avoid confusion with the raw radiographs, they are referred to as the "sinogram" in the following irrespective of the number of angles $\theta$.

In the same spirit as DVC, the displacement field is obtained from the minimization of the In the same spirit as DVC, the displacement field is obtained from the minimization of the quadratic difference between the (re-)projected reference $3 \mathrm{D}$ image corrected by the displacement field $\Pi_{k}[f(\boldsymbol{x}-\boldsymbol{u}(\boldsymbol{x}, t))]$ and the sinogram in the deformed states at time $t$ captured at just a few angles $s\left(\boldsymbol{r}, \theta_{k}, t\right)$

$$
\chi_{\boldsymbol{u}}^{2}(t)=\frac{1}{N_{\theta}|\Xi| \gamma_{s}^{2}} \sum_{k, \boldsymbol{r}}\left(\Pi_{k}[f(\boldsymbol{x}-\boldsymbol{u}(\boldsymbol{x}, t))]-s\left(\boldsymbol{r}, \theta_{k}, t\right)\right)^{2}
$$

where the double sum over $(k, r)$ is the discrete integration over all pixels $\boldsymbol{r} \in \Xi$ at all projection angles $\theta_{k} \cdot \gamma_{s}^{2}$ is the variance of the sinogram noise, and $|\Xi|$ is the area (number of pixels) of the detector (or its utilized part). At this step, the displacement field may be expressed on the reduced basis as previously defined. Minimization of the functional leads to the displacement field from the nodal values

$$
\boldsymbol{u}_{i}(t)=\operatorname{Argmin}_{\boldsymbol{u}_{i}}\left(\chi_{\boldsymbol{u}}^{2}(t)\right)
$$

A Newton algorithm may be used to solve this problem iteratively using progressive correction of the displacement field, which is a correction obtained from the tangent linear problem about the current point. Because the correction displacement field, $\delta \boldsymbol{u}_{i}$, is in the range of small perturbations, the integrand in Eq. 4 for the reference image corrected by this displacement field can be expressed as

$$
\Pi_{k}\left[f\left(\boldsymbol{x}-\boldsymbol{u}_{i}(t) \Phi_{i}(\boldsymbol{x})\right)\right] \approx \Pi_{k}\left[f(\boldsymbol{x}-\boldsymbol{u}(t))-\delta \boldsymbol{u}_{i}(t) \Phi_{i}(\boldsymbol{x}) \boldsymbol{\nabla} f(\boldsymbol{x}-\boldsymbol{u}(t))\right] .
$$

Finally the linear system that has to be solved is

$$
\delta \boldsymbol{u}_{i}=\boldsymbol{N}_{i j}^{-1} \boldsymbol{n}_{j}
$$

with $\boldsymbol{N}$ the Hessian matrix and $n$ the second member vector based on the residual field,

$$
\begin{gathered}
\boldsymbol{N}_{i j}=\sum_{\theta_{k}, \boldsymbol{r}} \Pi_{k}\left[\Phi_{i}(\boldsymbol{x}) \boldsymbol{\nabla} f(\boldsymbol{x}-\boldsymbol{u}(t))\right] \Pi_{k}\left[\Phi_{j}(\boldsymbol{x}) \boldsymbol{\nabla} f(\boldsymbol{x}-\boldsymbol{u}(t))\right] \\
\boldsymbol{n}_{i}=\sum_{\theta_{k}, \boldsymbol{r}}\left(s\left(\boldsymbol{r}, \theta_{k}, t\right)-\Pi_{k}[f(\boldsymbol{x}-\boldsymbol{u}(t))]\right) \Pi_{k}\left[\Phi_{i}(\boldsymbol{x}) \boldsymbol{\nabla} f(\boldsymbol{x}-\boldsymbol{u}(t))\right]
\end{gathered}
$$


As in DVC, the residual field at convergence, $\rho\left(\boldsymbol{r}, \theta_{k}, t\right) \equiv\left(s\left(\boldsymbol{r}, \theta_{k}, t\right)-\Pi_{k}[f(\boldsymbol{x}-\right.$ $\boldsymbol{u}(t))]$, gives very precious information about the quality of the solution. However, in P-DVC, the residual fields $\left(N_{\theta} 2 \mathrm{D}\right.$ images) are in the projected domain (parameterized by $\boldsymbol{r}$ ). They are also affected by acquisition noise and artifacts, reconstruction and projection inaccuracies and mesh discretization errors (on a crack path, for example).

Let us emphasize here a very attractive property of this approach. Because computations operate in the projection space, acquisition artifacts can be corrected, and noise can be accounted for. Noise, for instance, is close to being white and Gaussian, which legitimates the use of a simple L2 norm of the projection residuals. Some additional artifacts can also be characterized (dead detector pixels, beam hardening, etc.), and hence, accounting for these features is feasible. It is fair to add that some artifacts can be corrected for in a pre-processing stage, leading to enhanced quality of the reconstruction. However, it should also be emphasized that for the present application, more than just the reconstructed volume, i.e., each individual projection, must be corrected, and this is very demanding. This contrasts considerably with the classical DVC where, even after a pre-processing of the radiographs to erase some artifacts, noise and uncorrected or partially corrected artifacts have been processed in the reconstruction; hence, spatial correlations have been built, and the spatial stationarity is broken. In this case, a complex metric (inverse of the covariance matrix) should be used in DVC to compute the theoretically correct cost functional. This is so complex that no one ever considers such a comparison metric in practice, and hence, registration is sub-optimal.

\subsection{Integrated P-DVC}

Aiming to reduce the number of degrees of freedom, another mechanical regularization can be introduced. The displacement field can be expressed as a linear combination of the fields obtained from a finite element computation based on the same meshed domain by exploiting a constitutive law. The kinematics are then controlled by fewer degrees of freedom, $p_{i}$, for example, those parameterizing the boundary conditions of the test or a specimen geometry including, for example, a crack front position.

Depending on how the modeling (including the mechanical behavior) can be trusted, a small weight can be given to the regularization so that it acts as a mechanical low-pass filter [26, 23, or a large weight can be ascribed enforcing the mechanical behavior over the entire domain, which, thereby, drastically reduces the number of effective degrees of freedom and, hence, the computation time. In application, only the limit of an infinite weight will be considered so that a homogeneous elastic behavior is strictly prescribed. It is noteworthy that boundary conditions are understood here at large and may contain additional parameters, such as the position of the crack tip. Moreover, because the displacement fields are directly expressed in mechanically admissible fields, the present formulation allows for the identification of model parameters. In this framework, the selected unknown parameters, whether they be boundary conditions, geometry or constitutive parameters, can be considered altogether as the unknown $p_{i}$ with $1 \leq i \leq N$.

A similar descent method is selected as previously. However, as the expression of the new degrees of freedom (or their incremental change) can be obtained from 
the nodal displacements, the present formulation can be deduced from the previous formulation. Computing the sensitivity fields is required

$$
S_{i j}=\frac{\partial \boldsymbol{u}_{i}(\{p\})}{\partial p_{j}}
$$

The correction $\delta p_{i}$ to the current estimate of the parameters $\boldsymbol{p}$ reduces to

$$
\delta p_{i}=H_{i j}^{-1} h_{j}
$$

with a Hessian matrix $\boldsymbol{H}$ and the second member $\boldsymbol{h}$

$$
H_{i j}=S_{m i} \boldsymbol{N}_{m n} S_{n j} \quad \text { or } \quad \boldsymbol{H}=\boldsymbol{S}^{\top} \boldsymbol{N} \boldsymbol{S}
$$

and

$$
h_{j}=S_{i j} \boldsymbol{n}_{i} \quad \text { or } \quad \boldsymbol{h}=\boldsymbol{S}^{\top} \cdot \boldsymbol{n}
$$

After convergence, the residual fields are instrumental in evaluating the validity of the mechanical model and the choice of the boundary conditions. If deemed necessary, this choice can be revisited to better capture the kinematics.

\subsection{Use of load measurement}

The previous procedure only takes into account images. However, purely kinematic information cannot set a stress scale and, hence, cannot provide access parameters such as the Young's modulus for an elastic law. Therefore, it is useful to also take into account any available measurement data such as that of a load cell. With the previous integrated procedure, the load $F_{c}(t ; \boldsymbol{p})$ can be computed with any chosen set of parameters $\boldsymbol{p}$ and can be compared to the measured parameters $F_{m}(t)$.

An additional term can be added to the functional 27,28 to perform the minimization in displacement and force

$$
\chi^{2}(t)=\chi_{u}^{2}(t)+\chi_{F}^{2}(t)
$$

with the force functional

$$
\chi_{F}^{2}(t)=\frac{1}{\gamma_{F}^{2}}\left(F_{c}(t)-F_{m}(t)\right)^{2}
$$

where $\gamma_{F}^{2}$ is the noise variance of the load cell, (this formula assumes that only one measurement is being used).

Let us stress that the extensive quantities are the cost functions $\chi^{2}$ times the number of measurements they involve (provided they are statistically independent). Therefore, the joint minimization of two sets of measurements $A$ and $B$ should be based on

$$
\chi_{A \cup B}^{2}=\frac{N_{A} \chi_{A}^{2}+N_{B} \chi_{B}^{2}}{N_{A}+N_{B}}
$$

where $N_{A}$ (resp. $N_{B}$ ) is the number of measurements of $A$ (resp. $B$ ). This ensures that the expectation value of each $\chi^{2}$ is unity. Therefore, when combining different loading states, individual $\chi_{\boldsymbol{u}}^{2}$ and $\chi_{F}^{2}$ should be weighted by $N_{\theta}|\Xi|$ and 1 , respectively. When several $\left(N_{t}\right)$ loading states are considered, because each instant 
involves the same expression, both $N_{A}$ and $N_{B}$ are multiplied by $N_{t}$; hence, the above weighted means remain invariant, and $N_{t}$ can be ignored.

In the following, two strategies will be followed. In the first strategy, the measurements of displacement are first performed at each loading step independently, and then, from these measurements and the force values, the Young's modulus will be estimated. A second strategy consists of determining the forces and displacement jointly with the functional defined above, that is, coupling different kinematic measurement when no damage is expected.

In the following test case, an elastic law will be used. The Young's modulus, $E$, can be easily identified considering all $N_{t}$ loading steps in a spatio-temporal framework 29]. This consists of minimizing the total functional (summed over time) with respect to the unknown parameter. Because the force is proportional to $E$, the force can be computed with a unitary Young's modulus, $F_{u}(t)$, and hence, $F_{c}(t)=E . F_{u}(t)$. Minimizing the total cost function with respect to $E$ thus leads simply to

$$
\sum_{t}\left(F_{m}(t)-E F_{u}(t)\right) F_{u}(t)=0
$$

and hence

$$
E=\frac{\sum_{t} F_{u}(t) F_{m}(t)}{\sum_{t} F_{u}(t)^{2}}
$$

\section{Case study}

\subsection{DCDC test}

The Double Cleavage Drilled Compression (DCDC) test refers to a parallelepipedic shaped specimen with a square cross section normal to the sample length axis and a centered cylindrical hole drilled in the center of one of the lateral faces. The specimen is subjected to a compressive load in the length direction (perpendicular to the hole axis). This type of test permits studying the fracture of brittle materials (originally used for studying crack propagation in glass 30,31]). Under compression, due to the geometry, a tensile stress concentration is generated around the central hole and initiates two symmetric mode I cracks that propagate on the mid-plane of the sample while the load increases [32, 33,34 .

\subsection{Test case presentation}

The DCDC specimen used in the current study is a $8.40 \times 8.40 \times 21.79 \mathrm{~mm}^{3}$ plaster sample with 1.4 vol.\% of copper powder (approximate size of $150 \mu \mathrm{m}$ ) to provide markers well distributed in the bulk with a sharp contrast for X-ray imaging (the X-ray absorption of copper $(\mathrm{Cu})$ is much greater than that of gypsum $\left.\left(\mathrm{CaSO}_{4}, 2 \mathrm{H}_{2} \mathrm{O}\right)\right)$. Two notches $(3.80 \mathrm{~mm}$ long from the center and $200 \mu \mathrm{m}$ thick) are introduced in the mold. Finally, a $2.30 \mathrm{~mm}$ diameter hole is drilled in the center.

The sample (figure 1(a)) is scanned in the LMT lab tomograph (cone beam, $149.6 \mathrm{kV}, 108.5 \mu \mathrm{A}, \mathrm{W}$ target) inside an in situ testing machine similar to the one designed by Buffière and Maire [35] (figure 1(b)). The voxel size at full resolution 


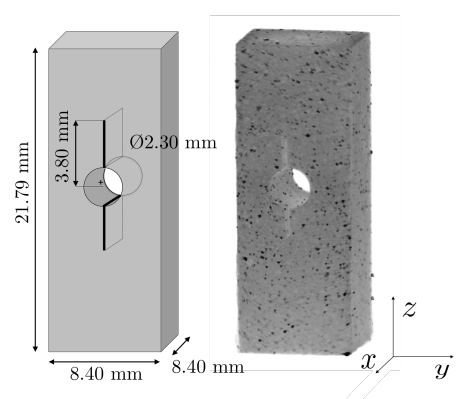

(a)

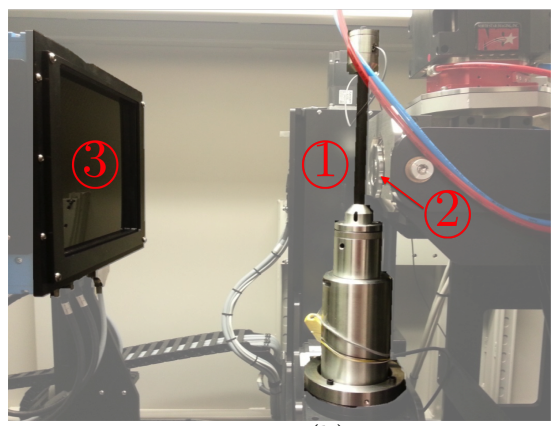

(b)

Fig. 1 (a) Drawing and 3D reconstruction of the sample, and (b) the in situ testing machine 35] inside the tomograph with (1), the testing machine with a carbon tube being supported by the rotating stage, (2), the X-ray source and (3), the detector. Note that the distance between the source and detector is not the one used in this article

was set to $15 \mu \mathrm{m}$. The complete scan of the reference state consisted of 1500 radiographs captured at equally spaced angles ranging over a $360^{\circ}$ revolution. One flat field was acquired after conditioning and before the experiment to perform a standard flat-field correction. One dark field was also acquired before the experiment. Because the reference volume was only acquired once, to enhance its quality and reduce the acquisition noise, each radiograph was an average over 50 projections. (This high number was found a posteriori to be unnecessary because the procedure was not sensitive to uncorrelated noise.) Finally, the entire reference scan (including storage time) requires $2.5 \mathrm{~h}$ to be completed.

\subsection{Mechanical test}

The in situ testing machine [35] is a tensile/compression machine controlled by the axial displacement of the lower part. The sample is placed on a composite tube that balances the load exerted on the specimen. The load cell capacity was $1 \mathrm{kN}$ with an uncertainty of $7 \mathrm{~N}$.

The experiment is composed of loading steps using a P-DVC approach (i.e., without acquisition and reconstruction of the deformed states). Previous works [21, 22,23. have shown that the procedure with a synchrotron beamline gave an accurate displacement field with 2 angles (chosen orthogonal). Similarly, angles for the P-DVC procedure are to be selected. A high sensitivity to the degrees of freedom allows for an accurate identification of the amplitude. The sensitivity to the crack position is selected to be maximized. Prior to the experiment, an elastic FE simulation with simple boundary conditions was performed on a synthetic gray level volume with a microstructure representative of that of the real sample, and the norm of the sensitivity $b_{j}$ was estimated for all integer angle values to select the most sensitive value. First, the angle $0^{\circ}$ (i.e., in the crack front direction) gave the greatest sensitivity due to an important orthogonal displacement around the crack. However, because of projection, some information (i.e., the displacement along the projection axis) was lost. Therefore, the second angle was selected at $90^{\circ}$ to provide the lacking sensitivity. 
Eight radiographs (acquired with an average of 50 projections) at each loading state were captured at every multiple of $45^{\circ}$ ( 8 were selected beforehand for caution, but only two were used in the following procedure, namely, at $0^{\circ}$ and $90^{\circ}$ ). The acquisition time for each of these steps was $35 \mathrm{~s}$. The test was composed of two parts:

- Seven loading steps without cracking (i.e., in an elastic regime), from 0 to $600 \mathrm{~N}$, spaced by approximately $100 \mathrm{~N}$;

- Six loading steps with crack initiation and propagation.

The fast 8 radiograph acquisitions were performed after an approximately 15 minute hold time to avoid possible relaxation phenomena. This procedure (again motivated by caution) was revealed to be unnecessary. At the end of the experiment, a final 3D scan (i.e., with a full acquisition of 1500 radiographs) was performed. This reconstruction was designed to track the possible non-planar crack propagation and for possible validation of the P-DVC results. Table 1 summarizes the main characteristics of each loading step.

Table 1 Loading step information for the two parts of the experiment

\begin{tabular}{|c|c|c|c|c|c|c|c|}
\hline Number & 1 & 2 & 3 & 4 & 5 & 6 & 7 \\
\hline full 3D scan & Yes & No & No & No & No & No & No \\
\hline load [N] & 0 & 91.8 & 188 & 305 & 393 & 488 & 609 \\
\hline visual crack & No & No & No & No & No & No & No \\
\hline
\end{tabular}

\begin{tabular}{|c|c|c|c|c|c|c|}
\hline Number & 8 & 9 & 10 & 11 & 12 & 13 \\
\hline full 3D scan & No & No & No & No & No & Yes \\
\hline load [N] & 628 & 638 & 651 & 669 & 691 & 625 \\
\hline visual crack & Yes & Yes & Yes & Yes & Yes & Yes \\
\hline
\end{tabular}

\subsection{Sinograms}

To control the reconstruction and the projection processes, the reconstruction of the reference state was performed using the ASTRA toolbox [36] and cone-beam projections. Because of the computational time and GPU memory requirement for ASTRA, the reconstruction from an FDK algorithm was carried out with different sinogram scales:

- Scale 2: Gaussian filtering of the sinogram with a characteristic length of 2 pixels followed by a down-sampling of 1 out of 2 pixels in both directions so that, at each angle, the sinogram was a $751 \times 972$ pixels image, and hence, after reconstruction, the effective voxel size was doubled to $30 \mu \mathrm{m}$. This scale was used for the reconstruction shown in fig. 11(a).

- Scale 4: Gaussian filtering of the sinogram with a characteristic length of 4 pixels followed by a down-sampling of 1 out of 4 pixels in both directions, resulting in $372 \times 486$ pixels images. This coarse scale was used to develop and initialize the finer scale.

The multi-scale procedure is applied on the deformed sinogram as well. 
As seen in figure 2, the specimen contains a large air bubble on one of the top corners. This bubble is meshed in the following finite element computation to account for its possible influence on the crack propagation.

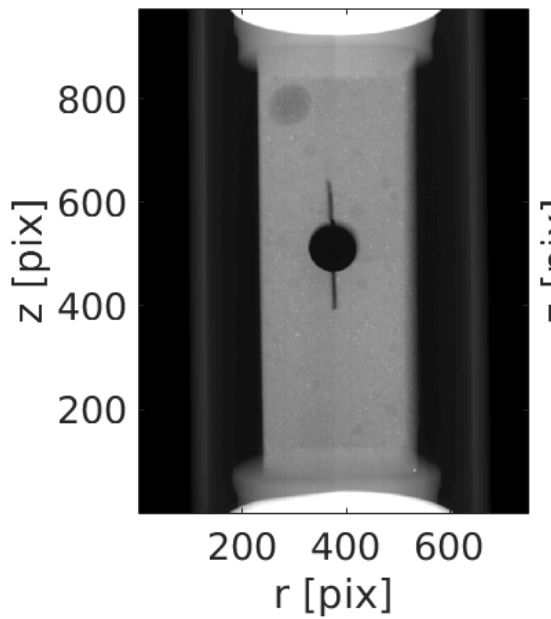

(a)

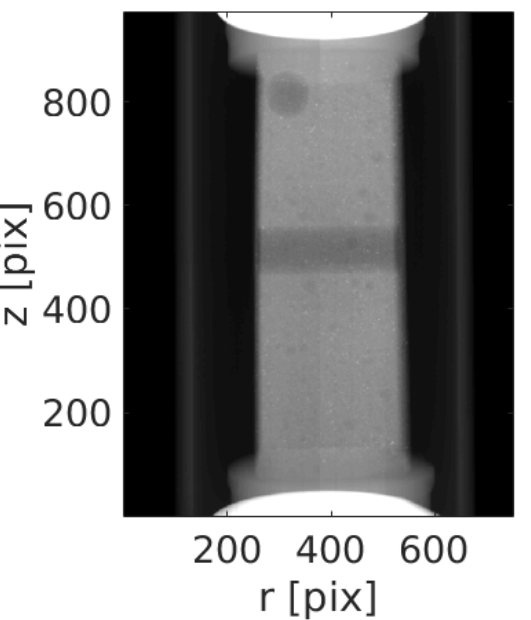

(b)

Fig. 2 Sinogram of the reference state at (a) $s\left(\boldsymbol{r}, \theta=0^{\circ}, t=1\right)$ and (b) $s\left(\boldsymbol{r}, \theta=90^{\circ}, t=1\right)$. $z$ is the rotation axis centered with respect to the image. One pixel $\rightarrow 30 \mu \mathrm{m}$

A bounding box around the sample (e.g., the white dashed rectangle in the projections in fig. 3) on the residual was selected to not bias the analysis with the still composite tube and to avoid top and bottom artifacts on the loading plates. Finally, the central hole and notches were hidden by a mask (delineated by full white lines) on the projections because of an important phase contrast. The studied area, which was inside the bounding box and excluded the central mask, was called the region of interest (ROI). The ROI is defined in $3 \mathrm{D}$, and its projection is considered on the sinogram (the exterior of the bounding box is considered to be void, but the excluded inner parts mask their entire shadow).

The sinogram and ROI for step 8 are shown in figure 3 . The crack may be visible at $\theta=0^{\circ}$ due to a phase contrast built along the optical path. This is contrasted with the following P-DVC procedure that estimates the position of the crack from the displacement field.

\subsection{Residual fields}

\subsubsection{Artifacts residual fields}

As shown in section 2.2 the P-DVC code minimizes the quadratic norm of the $2 \mathrm{D}$ residual fields $\rho\left(\boldsymbol{r}, \theta_{k}, t\right) \equiv s\left(\boldsymbol{r}, \theta_{k}, t\right)-\Pi_{k}[f(\boldsymbol{x}-\boldsymbol{u})]$. It is interesting to extend this procedure to the initial state $t=1$ where $\boldsymbol{u}$ is identically null per definition. 


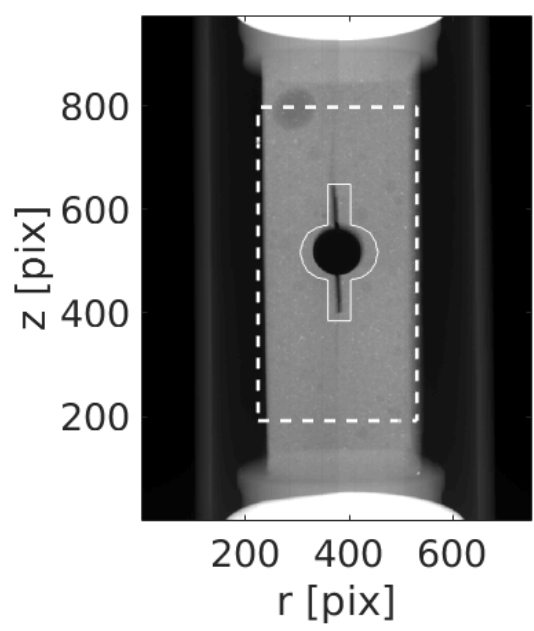

(a)

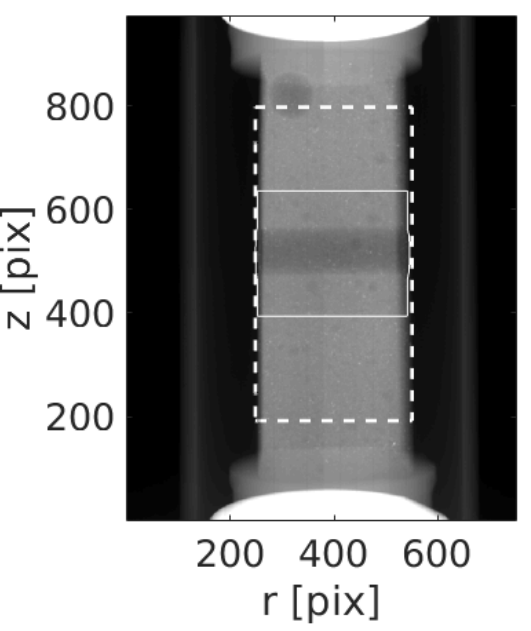

(b)

Fig. 3 Sinogram of the deformed specimen at (a) $s\left(\boldsymbol{r}, \theta=0^{\circ}, t=8\right)$ and (b) $s\left(\boldsymbol{r}, \theta=90^{\circ}, t=\right.$ $8)$. The vertical crack may be visible in (a) from the notches. The white dashed rectangle is the bounding box boundaries, and the full white lines are the central mask boundaries

Due to measurement noise and the slight inaccuracies of the reconstruction and re-projection algorithm, the artifact residual $\rho\left(\boldsymbol{r}, \theta_{k}, 1\right) \equiv s\left(\boldsymbol{r}, \theta_{k}, 0\right)-\Pi_{k}[f(\boldsymbol{x})]$ is not 0 . The initial artifact residual is shown in fig. 4 with a divergent color map to highlight differently positive and negative values. All residual fields are presented as a percent of the initial projection amplitude after removing the highest and lowest $5 \%$ of the gray levels. In this field, some features could be clearly interpreted as detector artifacts, and some are due to phenomena neglected in the reconstruction (beam hardening, phase contrast, and metal artifacts). This artifact residual field was observed to still be read in the following residuals $\rho\left(\boldsymbol{r}, \theta_{k}, t\right)$. Because $\rho\left(\boldsymbol{r}, \theta_{k}, 1\right)$ appears to be a limit that cannot be overstepped, in the following, we will focus on the difference $\Delta \rho\left(\boldsymbol{r}, \theta_{k}, t\right)=\rho\left(\boldsymbol{r}, \theta_{k}, t\right)-\rho\left(\boldsymbol{r}, \theta_{k}, 1\right)$ to minimize the effects of this initial systematic bias.

\subsubsection{Minimized residual fields}

$\Delta \rho\left(\boldsymbol{r}, \theta_{k}, t=8\right)$ is shown in figure 5 . The texture of this initial residual field displays a characteristic pattern of alternating positive and negative values along a preferential direction. This is the result of a displacement of the particles (vertical in this case). The crack path appears more clearly on the residual fields, but the exact position of the front is hardly visible.

A global criterion on the residual to evaluate the quality of the residual fields is the signal to noise ratio, which is defined as the logarithm of the quotient between the standard deviation of the projections and the residual fields,

$$
\operatorname{SNR}(t)=20 \log \left(\frac{\sigma(s)}{\sigma(\Delta \rho)}\right)
$$




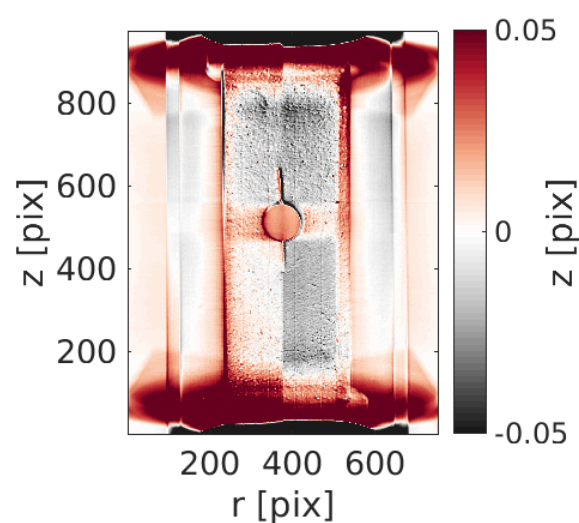

(a)

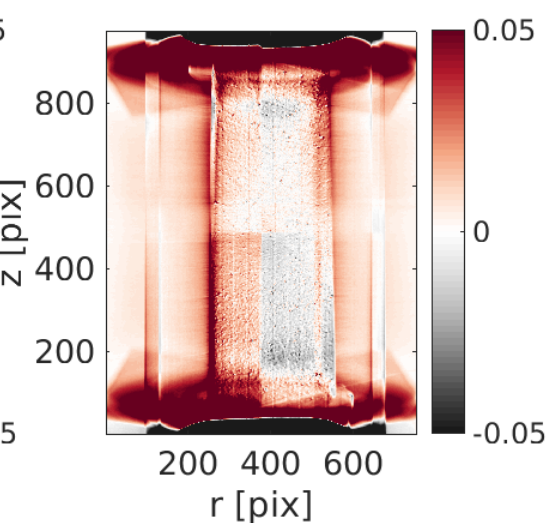

(b)

Fig. 4 Artifact residual (a) $\rho\left(\boldsymbol{r}, 0^{\circ}, 1\right)$ and (b) $\rho\left(\boldsymbol{r}, 90^{\circ}, 1\right)$. The four quadrants of the detector are visible

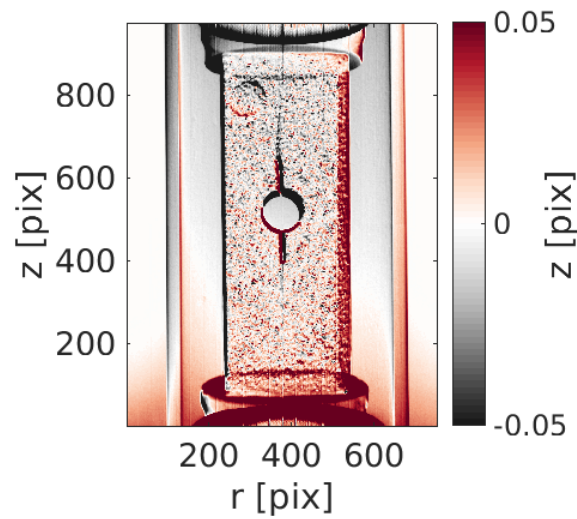

(a)

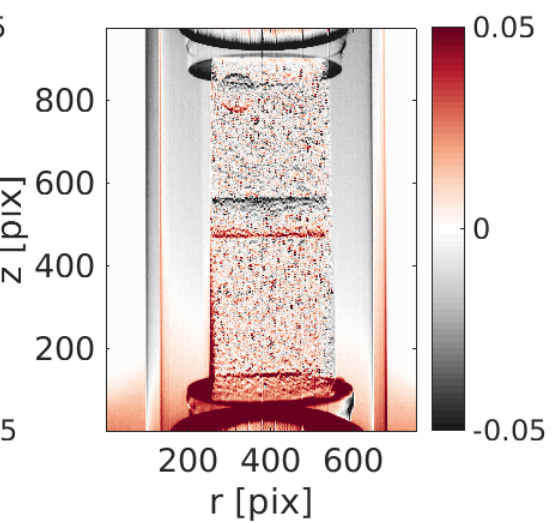

(b)

Fig. 5 Residual fields (a) $\Delta \rho\left(\boldsymbol{r}, 0^{\circ}, t=8\right)$ and (b) $\Delta \rho\left(\boldsymbol{r}, 90^{\circ}, t=8\right)$. The negative and positive patterns are the signature of a displacement

where the standard deviation is computed over the clipped and unmasked region of interest in the projection space.

\subsection{Mesh and regularization}

As previously explained, the displacement field is expressed as a combination of the fields related to the sensitivity to the selected parameters. These fields are computed based on a mechanical model, which is implemented with a finite element code. Because gypsum is an elastic brittle material, the selected mechanical behavior follows an elastic law (2 parameters: the unitary Young's modulus, $E=1 \mathrm{MPa}$, which is the conventional value, selected for identification purposes, and the Pois- 
son ratio, $\nu=0.2$ ) and a possible crack propagation that "splits" the volume into two parts.

According to the reconstruction of the deformed state in step 13, the crack path was observed to remain flat in the mid-plane of the sample. Hence, as shown in figure 6, the mesh was composed of two conforming parts that could be tightened (if there is no crack) with a common displacement on the facing nodes at the interface. The mesh was defined on the reference and deformed images. It had 1343 nodes and $4248 \mathrm{~T} 4$ elements. The large bubble observed in the corner was meshed, as it may affect the specimen stiffness. To not leave 'floating voxels' in the advection of the reference volume, the mesh was very slightly larger than the specimen volume. The effective cross-section of the mesh was approximately $4 \%$ larger than that of the volume itself.

The chosen parameters to control the kinematics are the following:

- Twelve degrees of freedom that represent the applied loading (i.e., 6 dofs at each of the top and bottom faces). These degrees of freedom describe a rigid body motion for each of these faces. Equivalently, they can be reorganized to describe the 6 degrees of freedom of the mean rigid body motion of the specimen, and the 6 others correspond to different elementary loadings: one tension and one torsion along the $z$ axis and two shear and two bending modes ( $x$ and $y$ axes). The sensitivity to the latter six boundary conditions were computed with a unitary displacement because of the linearity of the elastic law. These modes are shown in figure 6 .

- Two degrees of freedom for the crack front position. The crack was assumed to be in plane. The sensitivity for this parameter is the difference in the displacement field due to a crack propagating from a crack length of $p_{i}$ (with $i$ equal to 13 and 14 for the two crack fronts) to a slightly longer length $p_{i}+\mathrm{d} p_{i}$, which is normalized by the increment $\mathrm{d} p_{i}$.

\section{Results}

\subsection{Methodology}

The results of the P-DVC procedure are now presented. First, in Section 4.2 , the loading steps are considered independently to identify the displacement field boundary conditions and crack position. In this part, the first loading step where the crack propagated, step 8, has been selected to display the results in more details. In Section 4.3 , once the different displacement fields have been independently evaluated for each loading step, it is possible to identify the Young's modulus. Section 4.4 shows a refined evaluation of the displacement fields and crack position, taking into account the previously determined Young's modulus and the measured load. Finally, in Section 4.5 a minimum bound for the toughness is evaluated using the 7 th load step just prior to crack propagation. 


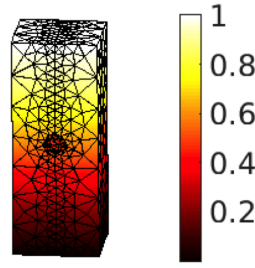

(a)

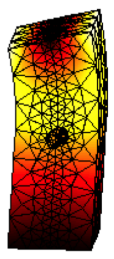

(d)

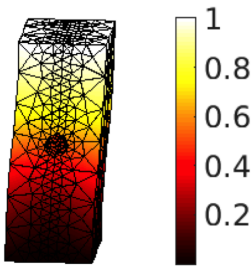

(b)

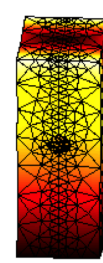

(e)

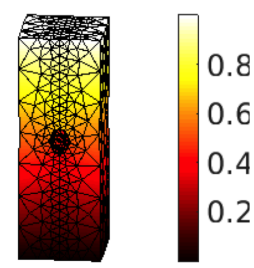

(c)

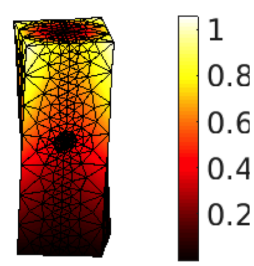

(f)

Fig. 6 T4 mesh adapted to the reconstruction for the six degrees of freedom applied on the upper face (while the bottom face is held fixed). On the first row: two shear and one tension modes; on the second row: two bending and one torsion modes. The color corresponds to the displacement amplitude

\subsection{Independent analysis of load steps}

The same analysis was performed for the 12 loading steps, and the entire series is presented for the global results, but when focusing on one loading step, the $8^{\text {th }}$ step is systematically selected since it is the step of crack inception.

The convergence criteria are defined on variations of both the SNR and the displacement field $\left(\Delta \mathrm{SNR}<10^{-3}\right.$ and $\|\Delta u(\boldsymbol{x}, t)\|_{2}<0.3 \mu \mathrm{m}$ or 0.01 voxels at scale 2), and the computation stops when both conditions are satisfied. The PDVC procedure was observed to converge in 4 to 6 iterations depending on the loading step, as shown in Figure 7 for step 8.
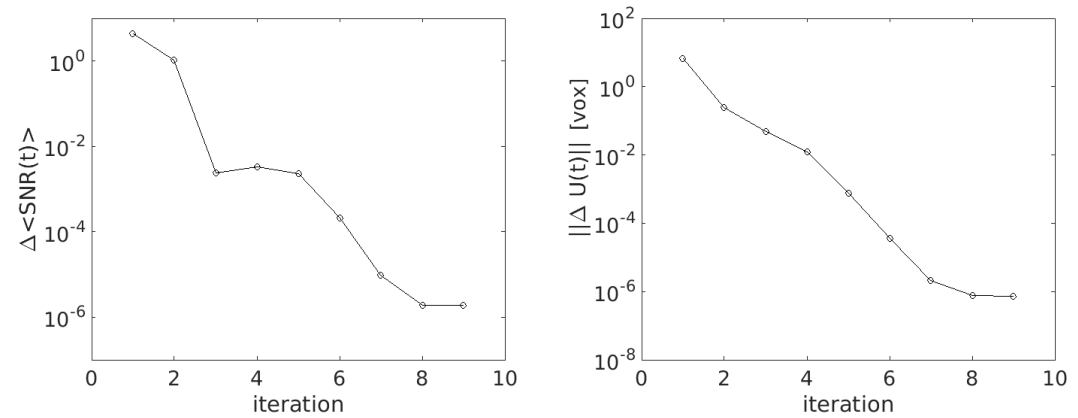

Fig. 7 Convergence of the variation of the mean SNR (left) and of the norm of the displacement variation (right) 
After convergence, the residual field for step 8 is shown in figure 8 The mean SNR ratios before and after correction of the displacement field were 16.49 and 21.89 , respectively. It can be seen that the residual field values were much smaller and smoother at the end of the process than in the initial state shown in figure 5 . The previous alternating positive and negative patterns (signaling a displacement) have been erased, confirming that the kinematics have been well captured. The crack path was much more apparent than the path from the initial residuals. Even the central part of the sample, which was masked in the ROI, was well corrected because of the regularization. Some horizontal and vertical lines are clearly visible. These lines correspond to detector artifacts that are not corrected by our procedure.

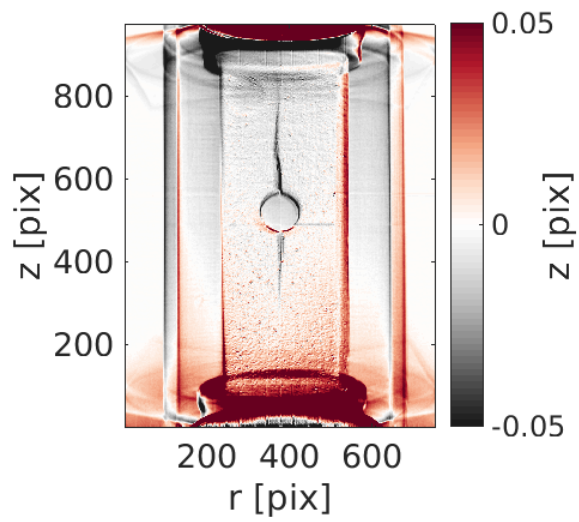

(a)

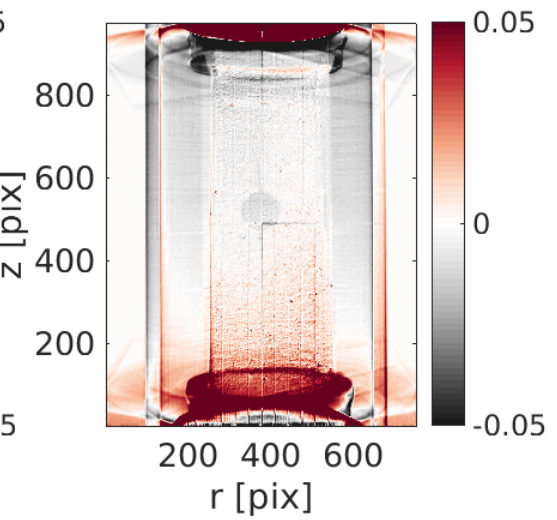

(b)

Fig. 8 Two-dimensional residual field at step 8 after the displacement field correction for (a) $\theta=0^{\circ}$ and (b) $90^{\circ}$. The black disk in the center of the right image is a reconstruction artifact

The displacement field in the three directions is shown figure 9. This displacement was composed of a large vertical rigid body motion of 4 voxels (due to the positioning of the sample and to the low rigidity of the testing machine), a compressive state and a bending on the upper face. This bending may be the cause of the non-parallelism of the top and bottom faces of the sample. The obtained displacement field was not a trivial compressive state and justified a full field measurement.

The two crack front positions were two degrees of freedom of our identification. At step 8, they indicate that both the top and bottom cracks propagated up to the top and bottom faces of the sample. This was unexpected, as the DCDC geometry should lead to a stable propagation for a displacement control, but the low rigidity testing machine may be responsible for releasing additional energy at the onset of propagation.

P-DVC computed at step 7 led to no crack inception, (i.e., no further than the notch), and indeed, in the residual before and after convergence, as shown in figure 10, no trace of a crack was apparent, in contrast to step 8. This observation is interesting because it provides a lower bound of the material toughness (see 


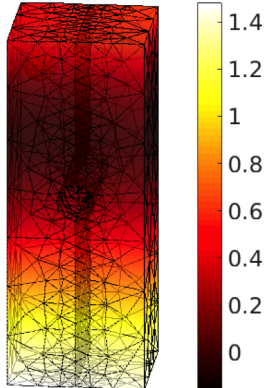

(a)
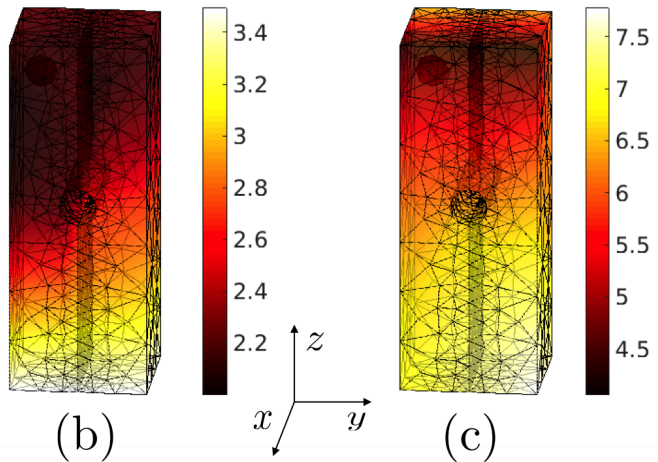

Fig. 9 Displacement field at convergence in voxels between the reference and step 8 in the (a) X, (b) Y and (c) Z direction. The voxel size was $30 \mu \mathrm{m}$.

Section 4.5. Moreover, the load in step 7 was only $19 \mathrm{~N}$ less than the load in step 8.

Other loading steps give similar results for the improvement of the residual fields. The SNR ratio before and after the procedure for each step is shown in figure 11. As expected, the initial SNR decreases with the loading step as the load and displacement increase. Even the initial residual at step 2 can be well improved, although it mostly corresponds to a rigid body motion.

It can be seen that after crack propagation, at steps 11 to 13, the SNR was less than before. With the reconstruction of the final 3D volume (step 13), one may see many cracks due to damage at the top and bottom ends of the sample as well as flexure cracks transverse to the sample axis at the position of the central hole. Such damages were not described in our modeling and hence induce a degradation of the registration quality, and the SNR decreases.

\subsection{Identification of elastic properties}

The Young's modulus identification was performed with a space-time regularization considering all previously analyzed loading steps. The measured forces were compared to the computed forces with convergence of the 14 kinematic parameters. As shown previously, the first 7 steps were considered to be in the elastic regime (no crack propagation); hence, they should give an accurate estimate of $E$ without significant model error. The following steps (including the through crack) should be amenable to a similar elastic analysis until significant further damage appears. Exploring different ranges for such an analysis led to the conclusion that, beyond step 11, the elastic model was not appropriate, and hence, the Young's modulus was estimated based on the first 10 loading steps (including the computed crack extension). From Eq. 18 the estimate was $E=1.12 \mathrm{GPa}$. This value was low compared to the literature [37,38] (2 to $5 \mathrm{GPa}$ ) but corresponds to the ex situ pre-tests on the same plaster and geometry (three tests with Young's moduli of $[998,931,940] \mathrm{MPa})$. A high water/plaster ratio was selected for easy molding of the specimen. Inserting this value back into the elastic model provides a com- 


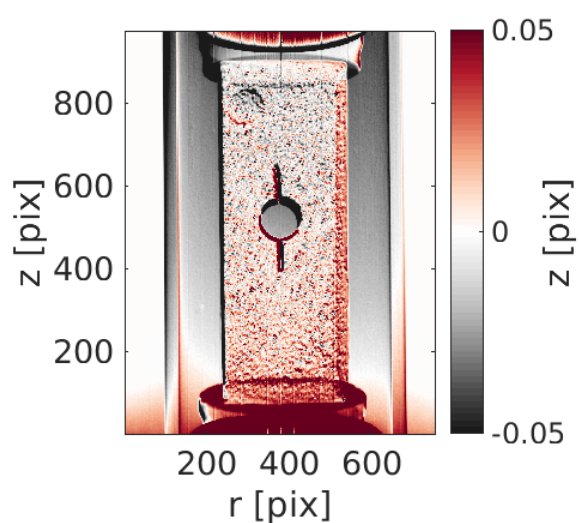

(a)

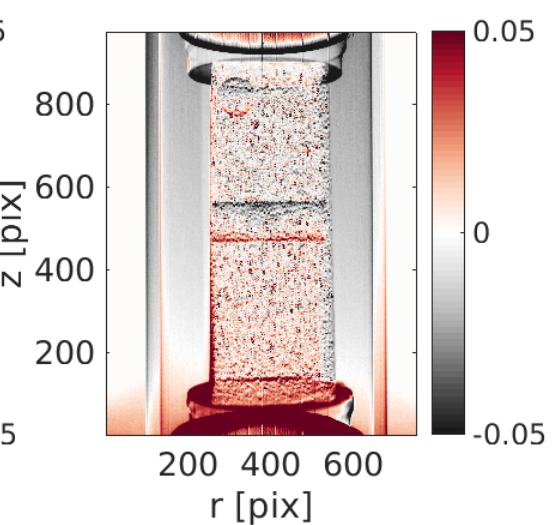

(b)

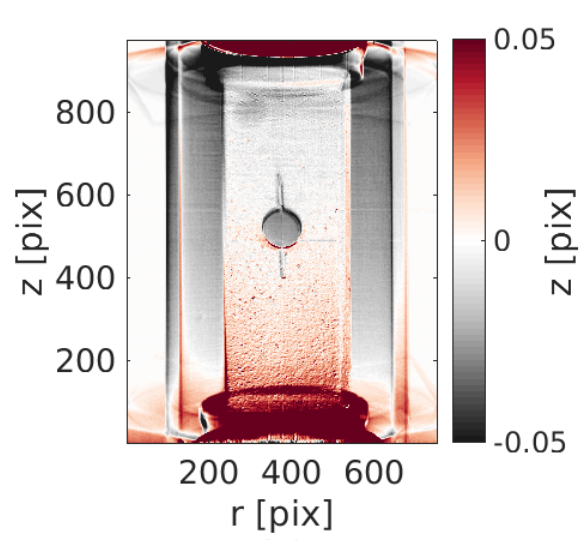

(c)

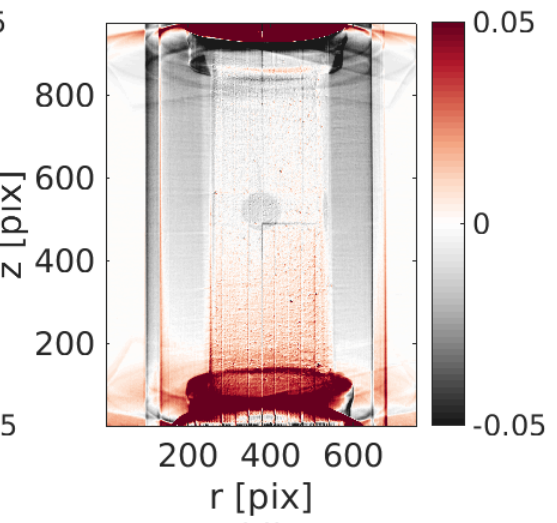

(d)

Fig. 10 Two-dimensional residual field at step 7 before the crack initiation for (a-c) $\theta=0^{\circ}$ and (b-d) $90^{\circ}$. The first line was prior to the displacement field correction, and the second was after. The vertical lines that are apparent in (c-d) are a detector artifact (also noticeable at step 8)

puted force that can be compared to the measured force, as shown in figure 12 . The Young modulus uncertainty was $1.5 \%$ as a result of that of the force.

4.4 Further regularization using identified elastic property and force signal

Once the Young's modulus was identified from all loading steps, it was possible to update the displacement field considering, in the P-DVC procedure, the additional constraint given by the force measurement (Eq. 14). This new $4 \mathrm{D}$ measurement gives a revised displacement field that can be compared to the previous displacement field. A comparison of the norm of the nodal displacements for the first 10 steps is shown in figure 13 . The maximum difference, $0.3 \%$, was small, meaning that the kinematics were well captured even prior to accounting for the force min- 


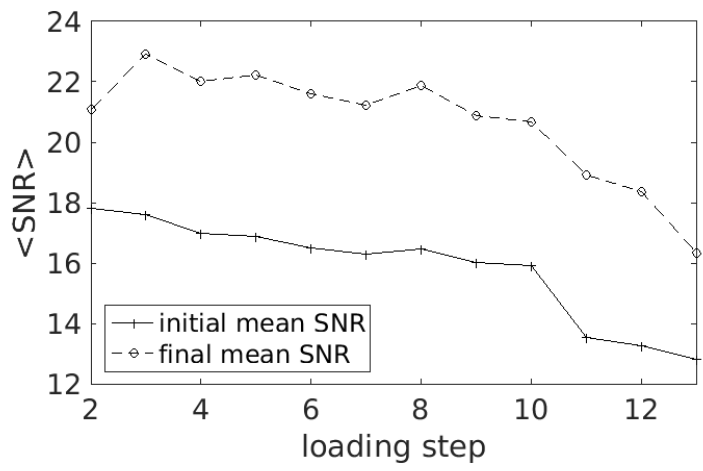

Fig. 11 Mean SNR before and after the displacement field correction for all loading steps

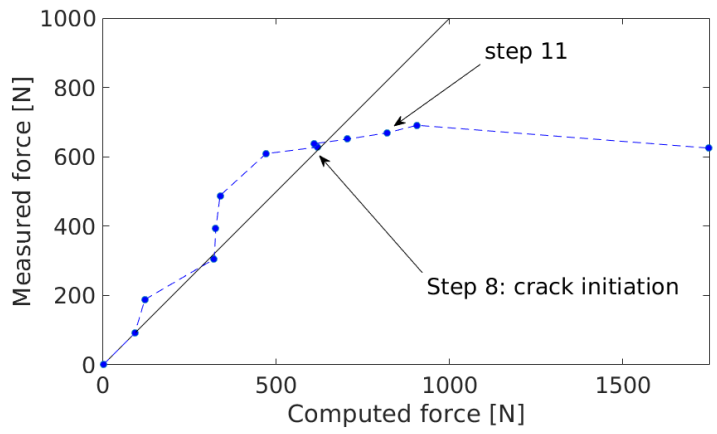

Fig. 12 Comparison between the measured and computed force considering the identified Young's modulus for the 13 steps. From step 11 and on, (and possibly earlier), the occurrence of damage was believed to make the comparison irrelevant

imization. A small difference was visible in the last 2 steps, considered likely due to less accurate model assumptions.

\subsection{Toughness evaluation}

Because step 8 corresponds to crack inception, the analysis of step 7 provides a lower bound for the sample toughness. In the same spirit, the loading of step 8 applied to a non-cracked specimen results in a higher bound of the toughness. Moreover, step 7 is only $19 \mathrm{~N}$ less than step 8 , and the crack geometry is known (notches); hence, the bounds should be accurate. The $G_{f}$ parameter was obtained with an elastic computation performed in Abaqus ${ }^{\circledR}$ using the J-integral method. The two bounds were $G_{f}=[10.4,11.1] \mathrm{J} / \mathrm{m}^{2}$.

This result can be compared with the standard $G_{f}$ measurements performed without full field analysis. In these standard procedures, only the compressive force was considered (hence, the displacement field was assumed to be in a uniform com- 


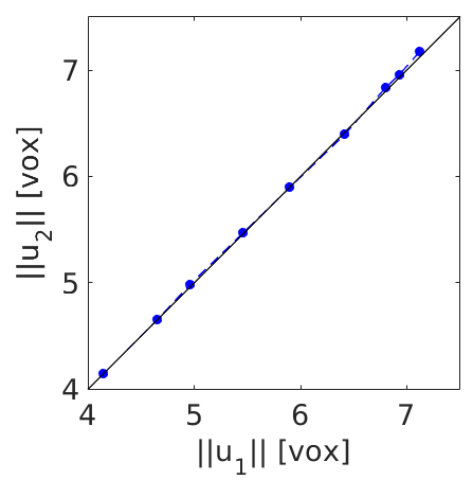

Fig. 13 Norm of the displacement field with the kinematic minimization only, $\left\|u_{1}\right\|$, and with the force and kinematic minimization (including the identified Young's modulus), $\left\|u_{2}\right\|$, expressed in voxels

pressive state). In our experiment, due to an important bending of the upper face, it was important to consider full field measurements. When the standard formula (ignoring bending) [39] was applied in the present case, $G_{f}=[9.5,10.1] \mathrm{J} / \mathrm{m}^{2}$, corresponding to a $9 \%$ difference with the previous evaluation.

The values of $G_{f}$ were high compared to the literature [37,38]. However, the low value of the identified Young's modulus gives $K_{I c}=[0.108,0.111] \mathrm{MPa} \mathrm{m}^{1 / 2}$, which is in the low range but compatible with literature values ranging from $K_{I c} \approx$ 0.1 to $0.5 \mathrm{MPa}^{1 / 2}$.

\section{Discussion}

This study was a first attempt to exploit a complete in situ mechanical test using $\mathrm{P}-\mathrm{DVC}$ in a lab tomograph. This method was $4 \mathrm{D}$ in the sense that one has access to the entire displacement field, $u(\boldsymbol{x}, t)$, in space and time at each loading step. The fact that only two projections (per step) were required endows the methodology with a much enhanced time resolution by a factor of several hundreds.

This opens new horizons for biological tissues where a low dose of X-ray radiation is required to limit radiation damage. However, one should emphasize that it is necessary that a reference volume has been reconstructed. Saving on X-ray dose only comes from the subsequent tracking of the motion in time where the additional dose is very small. Such a property may be used to add supplementary data at a low additional cost.

This also allows consideration of the time-dependent phenomena that could not be captured without P-DVC prosaically because of the evolution of the sample during the scan time (several hours or more). This may prevent a proper tomographic reconstruction and hence preclude the usage of volume correlation (classical DVC) to track the motion or any other evolution of the sample. Hence, on this ground, the superiority of P-DVC over DVC is obvious, as it makes studying situations that were simply out of reach when using classical means possible.

Complex specimen shapes or microstructures can be handled using this technique without restrictions. It should also be emphasized that when the geometry 
of the sample evolves during the test (as in the present case where the crack was not present in the initial geometry but appeared during the test), one may resort to the final state to extract a very precise geometry. More generally, possible nonplanar cracks could be considered in their final geometry, and cohesive elements could be implemented in the model to progressively open the crack, as indicated by the studied projections.

In the present study, a simple elastic model was shown to be appropriate (as judged from the residuals). In the case of more complex mechanical behavior measurement and identification, a more sophisticated model could be used. In the same spirit, as the crack propagation is a non-linear parameter, the finite element model and projected sensitivities may have to be re-computed when needed, possibly at each iteration of the procedure, which would increase the computation cost of the analysis.

Numerous artifacts are known to be detrimental to tomographic imaging. When working with raw projections, a number of possible corrections can be envisaged and tailored to the specific conditions of the acquisition of any given projection. In fact, reading projection residuals typically enables motion, intensity modulation, or other phenomena, whether localized in space or uniformly distributed, to be easily indicated and is required to reduce their level. Exploiting this flexibility allows for a very precise adjustment of the artifact corrections that can hardly be incorporated prior to reconstruction, as this would considerably increase the reconstruction time.

Noise is also a key feature often limiting the quality of reconstruction. Longrange correlations, which are present in the latter, are the result of the reconstruction that processes the noise. However, in the projections, noise is much less complex, and hence, taking it into account using a relevant weighting of the input data is easily accessible. This is expected to enhance the reliability and lower the uncertainty of the projection registration.

The limits of the proposed technique primarily come from our ability to model the test with a reduced set of parameters. It is essential that no (or very small) model error is introduced; otherwise, one may not be in a position to approach the actual projections from the deformation of the reference volume. The appropriateness of the model can be found in the fact that the residuals may be reduced to a low level that can be ascribed to noise (or to small amplitude artifacts that would not warrant corrections). Another potential limitation is the presence of large displacements that would violate the relevance of small perturbation analysis. Although a multiscale approach may help in correcting large amplitude motion, it is wise to perform the acquisition of projections at small loading increments.

In terms of the acquisition time, one may consider a much faster time lapse than the one used in the present study. The first 8 angles were captured, but only 2 were used. Moreover, a very long sequence (average over 50 projections) was acquired and is currently not the limiting factor for the proposed analysis. Ten projections were certainly sufficient, and two projections could be acquired within approximately one second. One limit here may be the time required to rotate the sample between the two views.

An appealing perspective to achieve a greater temporal resolution is to use time regularization to acquire a single radiograph at each loading step and perform a continuous rotating test. With such a $4 \mathrm{D}$ procedure, the unknowns contribute to the mechanical response of several steps, and with a suitable design, 
these unknowns can be determined with the entire time series, challenging the computational aspects of the inversion.

\section{Conclusion}

The analysis of a complete DCDC test with 13 loading steps was successfully performed and allowed for the analysis of the entire kinematics, the identification of the Young's modulus, and a bound on the critical elastic energy release rate. The identification of the Young's modulus has been performed considering all the loading steps at once, which is a specific feature made possible when using the 4D space and time proposed procedure.

This analysis was performed taking the actual boundary conditions of the test rather than assuming an ideal boundary condition, which would have led to a $9 \%$ error with respect to the toughness. In this measurement of a quasi-brittle sample, a simple elastic model with crack propagation was selected. This kinematic model has been validated because the low residuals showed that the kinematics were well captured. The kinematic regularization (based on only 14 parameters) was selected considering the studied sample and test. A scan of the cracked specimen at the end of the experiment allows the design of the mesh according to the real geometry.

This basis could be enriched by additional degrees of freedom, such as the shrinkage of the bottom and top parts due to a Poisson effect or a more complex crack path and front. However, phase contrast may induce specific features in the projection of the crack faces that are not currently modeled and that may limit the resolution of a much finer description.

This developed P-DVC procedure in a lab tomograph permits, from only one reference volume, the measurement of the displacement field of a "moving" specimen from two radiographs. P-DVC required an acquisition time 350 times less than that for standard DVC procedures. Further eliminating extraneous (unused) projections and performing fewer frame averages for radiographs would further increase this ratio well above three orders of magnitude.

\section{Acknowledgement}

This work has benefited from the support of the French "Agence Nationale de la Recherche" through the "Investissements d'avenir" Program under the reference "ANR-10-EQPX-37 MATMECA". We acknowledge Bumedijen Raka for his help in the ex situ pre-tests.

\section{References}

1. E. Maire. On the application of X-ray microtomography in the field of materials science. Advanced Engineering Materials, 3(8):539-546, 2001.

2. L. Salvo, P. Cloetens, E. Maire, S. Zabler, J.J. Blandin, J.Y. Buffière, W. Ludwig, E. Boller, D. Bellet, and C. Josserond. X-ray micro-tomography an attractive characterisation technique in materials science. Nuclear instruments and methods in physics research section B: Beam interactions with materials and atoms, 200:273-286, 2003. 
3. L. Salvo, M. Suéry, A. Marmottant, N. Limodin, and D. Bernard. 3D imaging in material science: Application of X-ray tomography. Comptes Rendus Physique, 11(9):641-649, 2010.

4. A. Guvenilir, T.M. Breunig, J.H. Kinney, and S.R. Stock. Direct observation of crack opening as a function of applied load in the interior of a notched tensile sample of AlLi 2090. Acta materialia, 45(5):1977-1987, 1997.

5. F. Beckmann, R. Grupp, A. Haibel, M. Huppmann, M. Nöthe, A. Pyzalla, W. Reimers, A. Schreyer, and R. Zettler. In-Situ Synchrotron X-Ray Microtomography Studies of Microstructure and Damage Evolution in Engineering Materials. Advanced Engineering Materials, 9(11):939-950, 2007.

6. E. Maire and P.J. Withers. Quantitative X-ray tomography. International materials reviews, 59(1):1-43, 2014.

7. H. Toda, E. Maire, S. Yamauchi, H. Tsuruta, T. Hiramatsu, and M. Kobayashi. In situ observation of ductile fracture using X-ray tomography technique. Acta Materialia, 59(5):1995-2008, 2011.

8. M. Vogelgesang, T. Farago, T.F. Morgeneyer, L. Helfen, T. dos Santos Rolo, A. Myagotin, and T. Baumbach. Real-time image-content-based beamline control for smart 4D X-ray imaging. Journal of Synchrotron Radiation, 23(5):1254-1263, 2016.

9. O. Ludwig, M. Dimichiel, L. Salvo, M. Suéry, and P. Falus. In-situ three-dimensional microstructural investigation of solidification of an Al-Cu alloy by ultrafast X-ray microtomography. Metallurgical and Materials Transactions A, 36(6):1515-1523, 2005.

10. K. Uesugi, M. Hoshino, A. Takeuchi, Y. Suzuki, and N. Yagi. Development of fast and high throughput tomography using CMOS image detector at SPring-8, 2012.

11. E. Maire, C. Le Bourlot, J. Adrien, A. Mortensen, and R. Mokso. $20 \mathrm{~Hz}$ X-ray tomography during an in situ tensile test. International Journal of Fracture, 200(1):3-12, 2016.

12. B.K. Bay, T.S. Smith, D.P. Fyhrie, and M. Saad. Digital volume correlation: threedimensional strain mapping using X-ray tomography. Experimental mechanics, 39(3):217226, 1999.

13. T.S. Smith, B.K. Bay, and M.M. Rashid. Digital volume correlation including rotational degrees of freedom during minimization. Experimental Mechanics, 42(3):272-278, 2002.

14. F. Hild, A. Bouterf, L. Chamoin, H. Leclerc, F. Mathieu, J. Neggers, F. Pled, Z. Tomičević, and S. Roux. Toward 4D Mechanical Correlation. Advanced Modeling and Simulation in Engineering Sciences, 3(1):17, May 2016.

15. A. Bouterf, J. Adrien, E. Maire, X. Brajer, F. Hild, and S. Roux. Failure Mechanisms of Plasterboard in Nail Pull Test Determined by X-ray Microtomography and Digital Volume Correlation. Experimental Mechanics, 56:1427-1437, 2016.

16. F. Prade, K. Fischer, D. Heinz, P. Meyer, J. Mohr, and F. Pfeiffer. Time resolved X-ray Dark-Field Tomography Revealing Water Transport in a Fresh Cement Sample. Scientific Reports, 6, 2016.

17. R.T. White, M. Najm, M. Dutta, F.P. Orfino, and E. Kjeang. Communication-Effect of Micro-XCT X-ray Exposure on the Performance of Polymer Electrolyte Fuel Cells. Journal of The Electrochemical Society, 163(10):F1206-F1208, 2016.

18. W. Hufenbach, R. Böhm, M. Gude, M. Berthel, A. Hornig, S. Ručevskis, and M. Andrich. A test device for damage characterisation of composites based on in situ computed tomography. Composites Science and Technology, 72(12):1361-1367, 2012.

19. H. Zhang, H. Toda, P.C. Qu, Y. Sakaguchi, M. Kobayashi, K. Uesugi, and Y. Suzuki. Three-dimensional fatigue crack growth behavior in an aluminum alloy investigated with in situ high-resolution synchrotron X-ray microtomography. Acta Materialia, 57(11):32873300, 2009.

20. J. Roth, J. Eller, and F.N. Büchi. Effects of synchrotron radiation on fuel cell materials. Journal of The Electrochemical Society, 159(8):F449-F455, 2012.

21. H. Leclerc, S. Roux, and F. Hild. Projection savings in CT-based digital volume correlation. Experimental Mechanics, 55(1):275-287, 2015.

22. T. Taillandier-Thomas, C. Jailin, S. Roux, and F. Hild. Measurement of 3D displacement fields from few tomographic projections. In SPIE Photonics Europe, page 98960. International Society for Optics and Photonics, 2016.

23. T. Taillandier-Thomas, S. Roux, and F. Hild. A soft route toward 4D tomography. Physical Review Letters, 117(2):025501, 2016.

24. M. H. Khalili. Tracking and modeling small motions at grain scale in granular materials under compression by X-Ray microtomography and discrete simulations. $\mathrm{PhD}$ thesis, Univ. Paris Est, 2016. 
25. S. Roux, F. Hild, P. Viot, and D. Bernard. Three-dimensional image correlation from X-ray computed tomography of solid foam. Composites Part A: Applied science and manufacturing, 39(8):1253-1265, 2008.

26. T. Taillandier-Thomas, S. Roux, T.F. Morgeneyer, and F. Hild. Localized strain field measurement on laminography data with mechanical regularization. Nuclear Instruments and Methods in Physics Research Section B: Beam Interactions with Materials and Atoms, 324:70-79, 2014.

27. F. Mathieu, H. Leclerc, F. Hild, and S. Roux. Estimation of Elastoplastic Parameters via Weighted FEMU and Integrated-DIC. Experimental Mechanics, 55(1):105-119, 2015.

28. M. Bertin, F. HILD, S. Roux, F. Mathieu, H. Leclerc, and P. Aimedieu. Integrated digital image correlation applied to elasto-plastic identification in a biaxial experiment. Journal of Strain Analysis for Engineering Design, 51(2):118-131, 2016.

29. G. Besnard, S. Guérard, S. Roux, and F. Hild. A space-time approach in digital image correlation: Movie-DIC. Optics and Lasers in Engineering, 49(1):71-81, 2011.

30. C. Janssen. Specimen for fracture mechanics studies on glass. Revue de Physique Appliquée, 12(5):803-803, 1977.

31. F. Célarié, S. Prades, D. Bonamy, L. Ferrero, E. Bouchaud, C. Guillot, and C. Marliere. Glass breaks like metal, but at the nanometer scale. Physical Review Letters, 90(7):075504, 2003.

32. T.A. Plaisted, A.V. Amirkhizi, and S. Nemat-Nasser. Compression-induced axial crack propagation in DCDC polymer samples: experiments and modeling. International journal of fracture, 141(3-4):447-457, 2006.

33. T. Fett, G. Rizzi, J.P. Guin, J.M. López-Cepero, and S.M. Wiederhorn. A fracture mechanics analysis of the double cleavage drilled compression test specimen. Engineering Fracture Mechanics, 76(7):921-934, 2009.

34. G. Pallares, L. Ponson, A. Grimaldi, M. George, G. Prevot, and M. Ciccotti. Crack opening profile in DCDC specimen. International journal of fracture, 156(1):11-20, 2009.

35. J.-Y. Buffière, E. Maire, J. Adrien, J.-P. Masse, and E. Boller. In situ experiments with X ray tomography: an attractive tool for experimental mechanics. Experimental mechanics, 50(3):289-305, 2010.

36. W. Van Aarle, W.J. Palenstijn, J. De Beenhouwer, T. Altantzis, S. Bals, K.J. Batenburg, and J. Sijbers. The ASTRA Toolbox: A platform for advanced algorithm development in electron tomography. Ultramicroscopy, 157:35-47, 2015.

37. P. Coquard and R. Boistelle. Thermodynamical approach to the brittle fracture of dry plasters. Journal of Materials Science, 31(17):4573-4580, 1996.

38. S. Meille. Etude du comportement mécanique du plâtre pris en relation avec sa microstructure. PhD thesis, INSA Lyon, 2010.

39. M.Y. He, M.R. Turner, and A.G. Evans. Analysis of the double cleavage drilled compression specimen for interface fracture energy measurements over a range of mode mixities. Acta Metallurgica et Materialia, 43(9):3453-3458, 1995. 